\title{
Relação das pessôas e instituições que doaram obras ou revistas á Biblioteca da Faculdade, durante o periodo compreendido entre 16 de abril e 15 de agosto de 1936:
}

Academia Colombiana de Juris-
prudencia de Bogotá. Alceu Toledo Piza Bellegarde.

Alcides Bezerra.

Alexandre Correia.

Alfredo Giribaldi Oddo.

Antonio da Fonseca Rosa.

Antonio Piccarolo.

Association Hongroise des Affaires Étrangères - Budapest.

Benjamin C. Oliveira.

Bibliografia Fascista - Roma.

Biblioteca Nacional de Buenos Aires.

Biblioteca da Assembléa Legislativa do Estado de São Paulo.

Biblioteca da Procuradoria Fiscal da Fazenda do Estado de São Paulo.

Candido Lobo.

Centro XI de Agosto.

Confederación Georgista Argentina.

Côrte de Apelação do Estado de Goyás.

Dalmo Belfort de Mattos.

Dardo A. Rietti.

Dario de Bittencourt.
Departamento de Cultura - La Habana.

Departamento Municipal de Cultura.

Diretoria de Organização e Defesa da produção.

Diretoria Geral de Informações, Estatistica e Divulgação — Rio de Janeiro.

Don José de Aguirre.

Editorial Reus, S. A. - Madrid.

Embaixada da Republica Argentina.

Eurico de Góes.

F. Domingues Carneiro.

Gabinete de Investigações de São Paulo.

Giorgio Del Vecchio.

Gremio de Cultura Mauá - Pelotas.

Helio Lobo.

Herminio Ferreira.

Ignacio Winisky.

Imprensa Metodista.

Imprensa Nacional - Rio de Janeiro.

Instituto da Ordem dos Advogados Brasileiros - Rio de Janeiro. 
Instituto de Filosofia de La Universidad de Córdoba.

Instituto de Organização Racional do Trabalho de São Paulo.

Instituto Paulista de Contabilidade.

Jamil Miguel Nami.

Javier Lopez Zavaleta.

João Rodrigues de Meréje.

Jorge Fonseca Junior.

Kenkokukinenjigiyo Kiokai.

Messias Junqueira.

Miguel Ramos Sucre.

Ministerio da Agricultura da Republica Argentina.

Ministerio das Relações Exteriores do Brasil.

Moniz Sodré.

Odilon Braga.

Osorio Dutra.

Paulo Martins.

Pedro Unzurrunzaga.

Pereira do Valle.
Philadelpho de Azevedo.

Prefeitura Municipal de Piracicaba.

Reitoria da Universidade do Rio de Janeiro.

René Thiollier.

Repartição de Estatistica e Arquivo do Estado.

Ricardo Levene.

Rodrigues Doria.

Salvador Barbalho Uchôa Cavalcanti.

Secretaria da Justiça do Estado de São Paulo.

Secretaria do Interior do Estado. do Rio Grande do Sul.

The Carnegie Foundation For The Advancement of Teaching.

Tito Rezende.

Universidad Nacional de Córdoba.

Universidad Nacional de Sureste - Merida/Mexico.

Verlag Von Julius Springer. 